\title{
BOUNDARY STABILIZATION OF MAXWELL'S EQUATIONS WITH SPACE-TIME VARIABLE COEFFICIENTS
}

\author{
Serge Nicaise ${ }^{1}$ And Cristina Pignotti ${ }^{2}$
}

\begin{abstract}
We consider the stabilization of Maxwell's equations with space-time variable coefficients in a bounded region with a smooth boundary by means of linear or nonlinear Silver-Müller boundary condition. This is based on some stability estimates that are obtained using the "standard" identity with multiplier and appropriate properties of the feedback. We deduce an explicit decay rate of the energy, for instance exponential, polynomial or logarithmic decays are available for appropriate feedbacks.
\end{abstract}

Mathematics Subject Classification. 93D15, 93B05, 93C20.

Received May 13, 2002.

\section{INTRODUCTION}

Let $\Omega$ be an open bounded domain in $\mathbb{R}^{3}$ with a boundary $\Gamma$ of class $C^{2}$. In this paper we study, under suitable boundary conditions, the stabilization of Maxwell's equations:

$$
\begin{aligned}
& D^{\prime}-\operatorname{curl}(\mu B)=0 \quad \text { in } \Omega \times(0,+\infty) \\
& B^{\prime}+\operatorname{curl}(\lambda D)=0 \quad \text { in } \Omega \times(0,+\infty) \\
& \operatorname{div} D=\operatorname{div} B=0 \quad \text { in } \Omega \times(0,+\infty) \\
& D(0)=D_{0} \text { and } B(0)=B_{0} \text { in } \Omega
\end{aligned}
$$

where $D, B$ are three-dimensional vector-valued functions of $t, x=\left(x_{1}, x_{2}, x_{3}\right) ; \mu=\mu(x, t), \lambda=\lambda(x, t)$ are scalar functions in $C^{1}(\bar{\Omega} \times[0,+\infty))$ uniformly bounded below by a positive constant and verifying suitable hypotheses; $D_{0}, B_{0}$ are the initial data in a suitable space.

Similarly to [15] we assume that a nonlinear Silver-Müller boundary condition holds

$$
g(x, D \times \nu) \times \nu+B \times \nu=0 \quad \text { on } \quad \Gamma \times(0,+\infty),
$$

\footnotetext{
Keywords and phrases. Maxwell's system, boundary stabilization.

${ }^{1}$ Université de Valenciennes et du Hainaut Cambrésis MACS, Institut des Sciences et Techniques de Valenciennes, 59313 Valenciennes Cedex 9, France; e-mail: s.nicaise@univ-valenciennes.fr

2 Dipartimento di Metodi e Modelli Matematici per le Scienze Applicate, Università di Roma "La Sapienza", Via A. Scarpa 16, 00161 Roma, Italy; e-mail: pignotti@dmmm.uniroma1.it 
where $\nu$ denotes the outward unit normal vector to $\Gamma$ and the mapping $g: \Gamma \times \mathbb{R}^{3} \rightarrow \mathbb{R}^{3}$ is assumed to be continuous and satisfying

$$
\begin{aligned}
& (g(x, E)-g(x, F)) \cdot(E-F) \geq 0, \forall E, F \in \mathbb{R}^{3}, x \in \Gamma \text { (monotonicity), } \\
& g(x, 0)=0, \forall x \in \Gamma, \\
& g(x, E) \cdot E \geq m_{1}|E|^{2}, \forall E \in \mathbb{R}^{3}:|E| \geq 1, x \in \Gamma \\
& |g(x, E)| \leq M(1+|E|), \forall E \in \mathbb{R}^{3}, x \in \Gamma
\end{aligned}
$$

for some positive constants $m_{1}, M$.

The linear case $g(x, D \times \nu)=\alpha(x) D \times \nu$ will retain a particular attention when $\alpha$ is a strictly positive function belonging to $C^{1}(\Gamma)$.

The above system with linear Silver-Müller boundary condition has retained the attention of many authors and stability results were obtained under different geometrical conditions in $[1,12,19]$ for $\lambda=\mu=1$.

For smooth coefficients $\lambda, \mu$ but time independent and $g$ not necessarily linear, explicit decay rates for solutions of the above system were recently given in $[5,15]$ when $\Omega$ is a connected domain with a smooth boundary $\Gamma$ consisting of a single connected component.

To our knowledge no stabilization result exists when the coefficients $\lambda$ and $\mu$ are time-dependent. Therefore the aim of this paper is to prove such results in the time-dependent case.

More precisely we give sufficient conditions which guarantee the decay of the energy of our system

$$
\mathcal{E}(t):=\frac{1}{2} \int_{\Omega}\left(\lambda(x, t)|D(x, t)|^{2}+\mu(x, t)|B(x, t)|^{2}\right) \mathrm{d} x .
$$

In the linear case we get exponential decay using the so-called identity with multiplier and standard arguments from [12]. In the nonlinear case contrary to $[5,15]$ we cannot use Liu's principle since our system is not reversible due to the time dependence of our coefficients, therefore we use a more direct method, namely we prove a stability estimate which is obtained with the help of the identity with multiplier and appropriate properties on $g$. By the new integral inequality from [5] we then deduce an explicit decay rate of the energy under appropriate assumptions on $g$.

We believe that the regularity assumption $\mu, \lambda \in C^{1}(\bar{\Omega} \times[0,+\infty))$ cannot be weakened since already for the wave equation with time independent coefficients, no controllability and stability results are available for less regular coefficients [2].

The paper is organized as follows: well-posedness of the problem is analysed in Section 2 under appropriate conditions on $\Omega, \lambda, \mu$ and $g$ using nonlinear semigroup theory. Section 3 is devoted to the proof of the identity with multiplier. We show in Section 4 the exponential stability of our system in the linear case, while Section 5 concerns stability results for general nonlinear feedbacks $g$.

\section{Well-POSEDNESS OF THE PROBLEM}

In this section we first show the well-posedness of problem (1.1-1.5) using nonlinear semigroup theory. We secondly establish the dissipativeness of the above system. To this end we introduce the Hilbert spaces (see e.g. $[13,16])$

$$
\begin{aligned}
& J(\Omega)=\left\{D \in L^{2}(\Omega)^{3} \mid \operatorname{div} D=0 \text { in } \Omega\right\}, \\
& \mathcal{H}=J(\Omega)^{2} .
\end{aligned}
$$

This last one is equipped with the time-depending norm induced by the time-depending inner product

$$
((D, B),(\tilde{D}, \tilde{B}))_{t}=\int_{\Omega}\{\lambda(x, t) D(x) \cdot \tilde{D}(x)+\mu(x, t) B(x) \cdot \tilde{B}(x)\} \mathrm{d} x, \forall(D, B),(\tilde{D}, \tilde{B}) \in \mathcal{H}
$$


Now define the (nonlinear) operator $A(t)$ from $\mathcal{H}$ into itself as follows:

$$
\begin{aligned}
D(A(t))= & \left\{(D, B) \in \mathcal{H} \mid \operatorname{curl}(\lambda D), \operatorname{curl}(\mu B) \in L^{2}(\Omega)^{3} ;\right. \\
& B \times \nu, D \times \nu \in L^{2}(\Gamma)^{3} \text { satisfying } \\
& B \times \nu+g(\cdot, D \times \nu) \times \nu=0 \text { on } \Gamma\}, \\
A(t)(D, B)= & (-\operatorname{curl}(\mu B), \operatorname{curl}(\lambda D)), \forall(D, B) \in D(A(t)) .
\end{aligned}
$$

Let us notice that the boundary condition (2.4) is meaningful due to the assumption (1.9) (see [15]).

We then see that formally the system (1.1-1.4) with boundary condition (1.5) is equivalent to

$$
\left\{\begin{array}{l}
\frac{\partial U}{\partial t}(t)+A(t) U(t)=0 \\
U(0)=U_{0}
\end{array}\right.
$$

when $U=(D, B)$ and $U_{0}=\left(D_{0}, B_{0}\right)$.

A general theory of such equations with linear operators $A(t)$ has been developed using semigroup theory in $[9,10,18]$ for instance.

For nonlinear operators $A(t)$ similar results exist (see $[3,6,8,14])$ but for maximal monotone operators $A(t)$ for one inner product independent of $t$.

For our system (2.6) we need a variant of such results for maximal monotone operators $A(t)$ for a timedependent inner product depending "smoothly" on $t$ (see Rem. 3 in [8]).

More precisely the next result holds whose proof is similar to the one in [8]:

Theorem 2.1. Let $X$ be a real separable Hilbert space. For a fixed $T>0$ and any $t \in[0, T]$ we assume that there exists an inner product $(\cdot, \cdot)_{t}$ on $X$ depending "smoothly" on $t$ in the following sense:

there exists $c>0$ such that

$$
\frac{\mathrm{d}}{\mathrm{d} t}(u, u)_{t} \leq 2 c(u, u)_{t}, \forall u \in X, t \in[0, T]
$$

Assume furthermore that:

(i) for all $t \in[0, T], A(t)$ is a maximal monotone operator for the inner product $(\cdot, \cdot)_{t}$;

(ii) the domain $D(A(t))=D$ of $A(t)$ is independent of $t$, for all $t \in[0, T]$;

(iii) there exists a positive constant $K$

such that

$$
\|A(t) u-A(s) u\|_{0} \leq K|t-s|\left(1+\|u\|_{0}+\|A(s) u\|_{0}\right), \forall u \in D, s, t \in[0, T] .
$$

Then for all $v \in D$ the evolution equation

$$
\left\{\begin{array}{l}
\frac{\partial u}{\partial t}(t)+A(t) u(t)=0, \text { for } 0 \leq t \leq T \\
u(0)=v
\end{array}\right.
$$

has a unique solution $u \in C([0, T] ; X)$ such that $u(t)$ belongs to $D$ for all $t \in[0, T]$, its strong derivative $\frac{\partial u}{\partial t}(t)=-A(t) u(t)$ exists and is continuous except at a countable numbers of values $t$.

Note that the condition (2.7) and Gronwall's inequality imply that

$$
\|u\|_{t} \leq \mathrm{e}^{c|t-s|}\|u\|_{s}, \forall u \in X, s, t \in[0, T] .
$$

This estimate implies in particular that the norms $\|\cdot\|_{t}$ are equivalent and gives the variation of the norm $\|\cdot\|_{t}$ with respect to $t$.

Remark 2.2. In the linear case the conditions (2.7) and $(i)$ to $($ iii $)$ imply that the triplet $\{A, X, D\}$ forms a CD-system in the sense of $[9,10]$. 
We shall now prove that the problem (2.6) has a unique solution by checking that (2.7) holds and that $A$ satisfies the assumptions (i) to (iii) with $X=\mathcal{H}$ under appropriate conditions on $\lambda$ and $\mu$.

We start with the time dependence of the inner product:

Lemma 2.3. Assume that $\lambda, \mu \in C^{1}(\bar{\Omega} \times[0,+\infty))$ are bounded below by a positive constant. Then for any $T>0$ there exists a constant $c$ (depending on $T$ ) such that (2.7) holds.

Proof. By a direct calculation we have

$$
\frac{\mathrm{d}}{\mathrm{d} t}\|U\|_{t}^{2}=\int_{\Omega}\left\{\frac{\partial \lambda}{\partial t}(x, t)|D(x)|^{2}+\frac{\partial \mu}{\partial t}(x, t)|B(x)|^{2}\right\} \mathrm{d} x,
$$

for $U=(D, B)$. By the assumptions on $\lambda$ and $\mu$, we obtain

$$
\frac{\mathrm{d}}{\mathrm{d} t}\|U\|_{t}^{2} \leq 2 c\|U\|_{t}^{2}
$$

with $2 c=\max \left\{\max _{(x, t) \in \bar{\Omega} \times[0, T]}\left|\frac{\partial \lambda}{\partial t}(x, t)\right| / \lambda(x, t) ; \max _{(x, t) \in \bar{\Omega} \times[0, T]}\left|\frac{\partial \mu}{\partial t}(x, t)\right| / \mu(x, t)\right\}$.

We now check the assumption (ii).

Lemma 2.4. Assume that $\lambda, \mu \in C^{1}(\bar{\Omega} \times[0,+\infty))$ are uniformly bounded below by a positive constant, that is

$$
\lambda(x, t) \geq L, \mu(x, t) \geq L, \forall x \in \Omega, t \in[0,+\infty),
$$

for some positive constant L. Then we have

$$
D(A(t))=\mathcal{H}_{1}, \forall t \geq 0,
$$

where we have set

$$
\begin{aligned}
\mathcal{H}_{1}= & \left\{(D, B) \in \mathcal{H} \mid \operatorname{curl} D, \operatorname{curl} B \in L^{2}(\Omega)^{3} ;\right. \\
& \left.B \times \nu, D \times \nu \in L^{2}(\Gamma)^{3} \text { satisfying }(2.4)\right\} .
\end{aligned}
$$

Proof. We use the standard formula

$$
\operatorname{curl}(\mu B)=\mu \operatorname{curl} B+\nabla \mu \times B .
$$

Therefore by the assumptions on $\mu$ we see that $B$ and $\operatorname{curl}(\mu B)$ belong to $L^{2}(\Omega)^{3}$ if and only if $B$ and $\operatorname{curl} B$ belong to $L^{2}(\Omega)^{3}$.

In the linear case we can even prove the

Lemma 2.5. If $\lambda, \mu \in C^{1}(\bar{\Omega} \times[0,+\infty))$ and $g(x, E)=\alpha(x) E$ with $\alpha \in C^{1}(\Gamma)$ such that $\nabla_{\Gamma} \alpha \in H^{1}(\Gamma)^{2}$, then we have

$$
D(A(t))=\mathcal{H}_{1}=\left\{(D, B) \in H^{1}(\Omega)^{6} \mid \operatorname{div} D=\operatorname{div} B=0, \nu \times(\alpha D \times \nu+B)=0\right\}, \forall t \geq 0 .
$$

Proof. As in [1], by the results from [17] for $(D, B)$ in $D(A(t)), \varphi=(D \times \nu) \times \nu$ belongs to

$$
W=\left\{\varphi \in H^{-1 / 2}(\Gamma)^{3}: \varphi \cdot \nu=0 \text { on } \Gamma \text { and } \operatorname{curl}_{\Gamma} \varphi \in H^{-1 / 2}(\Gamma)\right\} .
$$

Let us now show that $\alpha \varphi$ is also in $W$. 
Indeed by the identity

$$
\operatorname{curl}_{\Gamma}(\alpha \varphi)=\alpha \operatorname{curl}_{\Gamma} \varphi+\nabla_{\Gamma} \alpha \times \varphi
$$

we are reduced to show that each term of the above right-hand side belongs to $H^{-1 / 2}(\Gamma)$. This last result follows from the multiplication theorem in Sobolev spaces (see for instance Th. 1.4.4.2 of [7]) which yields that the product of an element from $H^{1}(\Gamma)$ with an element in $H^{-1 / 2}(\Gamma)$ is in $H^{-1 / 2}(\Gamma)$.

By the boundary condition (2.4), as in [1], this implies that

$$
\alpha(D \times \nu) \times \nu=-B \times \nu \in H^{1 / 2}(\Gamma)^{3},
$$

and therefore by standard regularity results [4] we obtain that $B$ belongs to $H^{1}(\Omega)^{3}$.

Again by the multiplication theorem in Sobolev spaces the product of an element from $H^{1}(\Gamma)$ with an element in $H^{1 / 2}(\Gamma)$ is in $H^{1 / 2}(\Gamma)$ and therefore the above identity yields $D \times \nu \in H^{1 / 2}(\Gamma)^{3}$ and therefore $D \in H^{1}(\Omega)^{3}$.

Let us pass to the hypothesis (i):

Lemma 2.6. Let $g$ satisfy the assumptions (1.6) to (1.8). Then $A(t)$ is a maximal monotone operator in $\mathcal{H}$ for the inner product $(\cdot, \cdot)_{t}$.

Proof. The proof is similar to the one in Lemma 2.5 of [15] and is left to the reader.

It remains to check the hypothesis (iii):

Lemma 2.7. If $\lambda, \mu \in C^{1}(\bar{\Omega} \times[0,+\infty))$ satisfy

$$
\frac{\partial \mu}{\partial t}(\cdot, t), \frac{\partial \lambda}{\partial t}(\cdot, t) \in C^{1}(\bar{\Omega}), \forall t \geq 0
$$

then (2.8) holds.

Proof. By the definition of $A(t)$ and the finite increment Theorem, we have

$$
\begin{aligned}
A(t) U-A(s) U= & (-\operatorname{curl}((\mu(t)-\mu(s)) B), \operatorname{curl}((\lambda(t)-\lambda(s)) D) \\
= & (t-s)\left(-\operatorname{curl}\left(\frac{\partial \mu}{\partial t}\left(s_{1}\right) B\right), \operatorname{curl}\left(\frac{\partial \lambda}{\partial t}\left(s_{2}\right) D\right)\right), \\
= & (t-s)\left(-\frac{\frac{\partial \mu}{\partial t}\left(s_{1}\right)}{\mu(s)} \operatorname{curl}(\mu(s) B)-\mu(s) \nabla\left(\frac{\frac{\partial \mu}{\partial t}\left(s_{1}\right)}{\mu(s)}\right) \times B,\right. \\
& \left.\frac{\frac{\partial \lambda}{\partial t}\left(s_{2}\right)}{\lambda(s)} \operatorname{curl}(\lambda(s) D)+\lambda(s) \nabla\left(\frac{\frac{\partial \lambda}{\partial t}\left(s_{2}\right)}{\lambda(s)}\right) \times D\right),
\end{aligned}
$$

for $U=(D, B) \in \mathcal{H}_{1}$ and some $s_{1}, s_{2} \in(s, t)$.

The assumptions on $\lambda$ and $\mu$ directly lead to the conclusion.

Theorem 2.1 allows to conclude the following existence result:

Corollary 2.8. Assume that $g$ satisfies the assumptions of Lemma 2.6 and that $\lambda, \mu \in C^{1}(\bar{\Omega} \times[0,+\infty))$ satisfy (2.11) and (2.12). Then for all $\left(D_{0}, B_{0}\right) \in \mathcal{H}_{1}$ and all $T>0$, the system (1.1-1.4) with boundary condition (1.5) admits a unique solution $(D, B) \in C([0, T] ; \mathcal{H})$ such that $(D(t), B(t))$ belongs to $\mathcal{H}_{1}$ for all $t \in[0, T]$ and their strong derivatives $\frac{\partial D}{\partial t}(t)=\operatorname{curl}(\mu(t) B(t))$ and $\frac{\partial B}{\partial t}(t)=-\operatorname{curl}(\lambda(t) D(t))$ exist and are continuous except at a countable numbers of values $t$.

We finish this section by analysing the dissipativity of our system. 
Lemma 2.9. Let $\left(D_{0}, B_{0}\right)$ be an initial pair in $\mathcal{H}_{1}$ and let $(D, B)$ be the solution of the system (1.1-1.4) with boundary condition (1.5). Then the derivative of the energy (defined by (1.10)) is

$$
\mathcal{E}^{\prime}(t)=\int_{\Gamma} \mu \lambda(D \times \nu) \cdot B \mathrm{~d} \Gamma+\frac{1}{2} \int_{\Omega}\left\{\frac{\partial \lambda}{\partial t}|D|^{2}+\frac{\partial \mu}{\partial t}|B|^{2}\right\} \mathrm{d} x, \quad t>0 .
$$

Proof. Deriving (1.10) we obtain

$$
\mathcal{E}^{\prime}=\frac{1}{2} \int_{\Omega}\left\{2 \lambda D \cdot D^{\prime}+2 \mu B \cdot B^{\prime}+\frac{\partial \lambda}{\partial t}|D|^{2}+\frac{\partial \mu}{\partial t}|B|^{2}\right\} \mathrm{d} x
$$

then, by (1.1) and (1.2),

$$
\mathcal{E}^{\prime}=\frac{1}{2} \int_{\Omega}\left\{2 \lambda D \cdot \operatorname{curl} \mu B-2 \mu B \cdot \operatorname{curl} \lambda D+\frac{\partial \lambda}{\partial t}|D|^{2}+\frac{\partial \mu}{\partial t}|B|^{2}\right\} \mathrm{d} x
$$

Therefore, integrating by parts we obtain

$$
\mathcal{E}^{\prime}=\int_{\Gamma} \mu \lambda(D \times \nu) \cdot B \mathrm{~d} \Gamma+\frac{1}{2} \int_{\Omega}\left\{\frac{\partial \lambda}{\partial t}|D|^{2}+\frac{\partial \mu}{\partial t}|B|^{2}\right\} \mathrm{d} x .
$$

Lemma 2.10. Let $\left(D_{0}, B_{0}\right)$ be an initial pair in $\mathcal{H}_{1}$ and let $(D, B)$ be the solution of the system (1.1-1.4) with boundary condition (1.5). Then for all $0 \leq S<T<+\infty$

$$
\mathcal{E}(S)-\mathcal{E}(T)=\int_{S}^{T} \int_{\Gamma} \mu \lambda g(x, D \times \nu) \cdot D \times \nu \mathrm{d} \Gamma \mathrm{d} t-\frac{1}{2} \int_{S}^{T} \int_{\Omega}\left\{\frac{\partial \lambda}{\partial t}|D|^{2}+\frac{\partial \mu}{\partial t}|B|^{2}\right\} \mathrm{d} x \mathrm{~d} t .
$$

Proof. The identity (2.14) directly follows from (2.13) since the boundary condition (1.5) implies that

$$
(D \times \nu) \cdot B=-D \cdot(B \times \nu)=D \cdot(g(\cdot, D \times \nu) \times \nu)=-g(\cdot, D \times \nu) \cdot D \times \nu
$$

In the linear case we have as an easy consequence

Corollary 2.11. Let $\left(D_{0}, B_{0}\right)$ be an initial pair $\in \mathcal{H}_{1}$ and let $(D, B)$ be the solution of the system (1.1-1.4) with boundary condition $(1.5)$ with $g(x, D)=\alpha(x) D$. Then,

$$
\begin{aligned}
& \mathcal{E}(S)-\mathcal{E}(T)=\int_{S}^{T} \int_{\Gamma} \mu \lambda \frac{1}{\alpha}\left|B_{\tau}\right|^{2} \mathrm{~d} \Gamma \mathrm{d} t-\frac{1}{2} \int_{S}^{T} \int_{\Omega}\left\{\frac{\partial \lambda}{\partial t}|D|^{2}+\frac{\partial \mu}{\partial t}|B|^{2}\right\} \mathrm{d} x \mathrm{~d} t \\
& \mathcal{E}(S)-\mathcal{E}(T)=\int_{S}^{T} \int_{\Gamma} \mu \lambda \alpha\left|D_{\tau}\right|^{2} \mathrm{~d} \Gamma \mathrm{d} t-\frac{1}{2} \int_{S}^{T} \int_{\Omega}\left\{\frac{\partial \lambda}{\partial t}|D|^{2}+\frac{\partial \mu}{\partial t}|B|^{2}\right\} \mathrm{d} x \mathrm{~d} t
\end{aligned}
$$

for all $0 \leq S<T<+\infty$, where $D_{\tau}$ and $B_{\tau}$ denote the tangential components of $D$ and $B$.

Remark 2.12. If $\lambda$ and $\mu$ are non-increasing in time, i.e.

$$
\frac{\partial \lambda}{\partial t} \leq 0, \quad \frac{\partial \mu}{\partial t} \leq 0, \quad \forall x \in \Omega, t \in(0,+\infty)
$$

then by Lemma 2.10, the energy $\mathcal{E}(\cdot)$ is non-increasing on $(0,+\infty)$ since $g(\cdot, D \times \nu) \cdot D \times \nu \geq 0$. 
Remark 2.13. We have just seen that the condition (2.17) is a sufficient condition for the nonincreaseness of the energy. Let us show by an example that this condition is a necessary condition for the nonincreaseness of the energy. For that purpose denote by $A_{0}$ the above operator $A$ with the choice $\lambda=\mu=1$ and $g(\cdot, D)=D$. Since its domain is equal to $H^{1}(\Omega)^{6}$ which is compactly embedded into $\mathcal{H}$, the operator $A_{0}$ is skew-symmetric with a discrete spectrum (see [4]).

Fix an eigenvector $(E, H)$ of $A_{0}$ of eigenvalue $i r$, with $r \neq 0$. Now take the system (1.1-1.4) and (1.5) with $\lambda(t)=\mu(t)$ but independent of $x$ such that $\mu \in C^{1}([0, \infty))$ and $g(\cdot, D)=D$.

Then one readily checks that the solution $(D, B)$ of that system is given by

$$
\begin{aligned}
& D(x, t)=\mathrm{e}^{-i r \int_{0}^{t} \mu(s) \mathrm{d} s} E(x), \\
& B(x, t)=\mathrm{e}^{-i r \int_{0}^{t} \mu(s) \mathrm{d} s} H(x) .
\end{aligned}
$$

The energy of that solution is given by

$$
\mathcal{E}(t)=c \mu(t)
$$

where $c=\frac{1}{2} \int_{\Omega}\left(|E(x)|^{2}+|H(x)|^{2}\right) \mathrm{d} x$ and consequently $\mathcal{E}^{\prime} \leq 0$ if and only if $\mu^{\prime} \leq 0$.

\section{An identity With MULtiPlieR}

This section is mainly devoted to the proof of the so-called identity with multiplier.

Lemma 3.1 is completely analogous to Lemma 2.2 in [20] that has extended to Maxwell's equations with space-variable coefficients a previous formula obtained by Komornik [12] for the case with constant coefficients. We give the proof for the reader's convenience.

Lemma 3.1. Take $\left(D_{0}, B_{0}\right) \in \mathcal{H}_{1}, q \in\left(C^{1}(\bar{\Omega})\right)^{3}$ and let $T>0$. Then the solution of (1.1-1.4) satisfies the identity

$$
\begin{aligned}
{\left[2 \int_{\Omega}(D \times B) \cdot q\right]_{0}^{T}=} & \int_{0}^{T} \int_{\Gamma}\left\{-\left(\lambda|D|^{2}+\mu|B|^{2}\right)(q \cdot \nu)+2 \mu(q \cdot B)(\nu \cdot B)+2 \lambda(q \cdot D)(\nu \cdot D)\right\} \mathrm{d} \Gamma \mathrm{d} t \\
& +\int_{0}^{T} \int_{\Omega}\left\{(\operatorname{div} q)\left(\lambda|D|^{2}+\mu|B|^{2}\right)-2 \sum_{i, k=1}^{3} \frac{\partial q_{i}}{\partial x_{k}}\left(\lambda D_{i} D_{k}+\mu B_{i} B_{k}\right)\right\} \mathrm{d} x \mathrm{~d} t \\
& +\int_{0}^{T} \int_{\Omega}\left\{-(q \cdot \nabla \lambda)|D|^{2}-(q \cdot \nabla \mu)|B|^{2}\right\} \mathrm{d} x \mathrm{~d} t
\end{aligned}
$$

where $D_{i}, B_{i}$ for $i=1,2,3$ are the scalar components of $D, B$.

Proof. By equations (1.1) and (1.2) we have in $\Omega \times(0,+\infty)$,

$$
\begin{aligned}
D_{1}^{\prime} & =\frac{\partial \mu}{\partial x_{2}} B_{3}-\frac{\partial \mu}{\partial x_{3}} B_{2}+\mu \frac{\partial B_{3}}{\partial x_{2}}-\mu \frac{\partial B_{2}}{\partial x_{3}}, \\
D_{2}^{\prime} & =\frac{\partial \mu}{\partial x_{3}} B_{1}-\frac{\partial \mu}{\partial x_{1}} B_{3}+\mu \frac{\partial B_{1}}{\partial x_{3}}-\mu \frac{\partial B_{3}}{\partial x_{1}}, \\
D_{3}^{\prime} & =\frac{\partial \mu}{\partial x_{1}} B_{2}-\frac{\partial \mu}{\partial x_{2}} B_{1}+\mu \frac{\partial B_{2}}{\partial x_{1}}-\mu \frac{\partial B_{1}}{\partial x_{2}}, \\
B_{1}^{\prime} & =\frac{\partial \lambda}{\partial x_{3}} D_{2}-\frac{\partial \lambda}{\partial x_{2}} D_{3}+\lambda \frac{\partial D_{2}}{\partial x_{3}}-\lambda \frac{\partial D_{3}}{\partial x_{2}}, \\
B_{2}^{\prime} & =\frac{\partial \lambda}{\partial x_{1}} D_{3}-\frac{\partial \lambda}{\partial x_{3}} D_{1}+\lambda \frac{\partial D_{3}}{\partial x_{1}}-\lambda \frac{\partial D_{1}}{\partial x_{3}}, \\
B_{3}^{\prime} & =\frac{\partial \lambda}{\partial x_{2}} D_{1}-\frac{\partial \lambda}{\partial x_{1}} D_{2}+\lambda \frac{\partial D_{1}}{\partial x_{2}}-\lambda \frac{\partial D_{2}}{\partial x_{1}} .
\end{aligned}
$$


Therefore

$$
\begin{aligned}
2\left(D_{1} B_{2} q_{3}\right)^{\prime}= & 2\left(\frac{\partial \lambda}{\partial x_{1}} D_{3}-\frac{\partial \lambda}{\partial x_{3}} D_{1}\right) D_{1} q_{3}+2\left(\frac{\partial \mu}{\partial x_{2}} B_{3}-\frac{\partial \mu}{\partial x_{3}} B_{2}\right) B_{2} q_{3} \\
& +2\left(\lambda \frac{\partial D_{3}}{\partial x_{1}}-\lambda \frac{\partial D_{1}}{\partial x_{3}}\right) D_{1} q_{3}+2\left(\mu \frac{\partial B_{3}}{\partial x_{2}}-\mu \frac{\partial B_{2}}{\partial x_{3}}\right) B_{2} q_{3} \\
= & 2 q_{3}\left(\frac{\partial \lambda}{\partial x_{1}} D_{3} D_{1}-\frac{\partial \lambda}{\partial x_{3}} D_{1}^{2}\right)+2 q_{3}\left(\frac{\partial \mu}{\partial x_{2}} B_{3} B_{2}-\frac{\partial \mu}{\partial x_{3}} B_{2}^{2}\right) \\
& +2 q_{3} \lambda \frac{\partial D_{3}}{\partial x_{1}} D_{1}-q_{3} \lambda \frac{\partial\left(D_{1}^{2}\right)}{\partial x_{3}}+2 q_{3} \mu \frac{\partial B_{3}}{\partial x_{2}} B_{2}-q_{3} \mu \frac{\partial\left(B_{2}^{2}\right)}{\partial x_{3}}
\end{aligned}
$$

and analogously

$$
\begin{aligned}
2\left(D_{2} B_{1} q_{3}\right)^{\prime}= & 2\left(\frac{\partial \lambda}{\partial x_{3}} D_{2}-\frac{\partial \lambda}{\partial x_{2}} D_{3}\right) D_{2} q_{3}+2\left(\frac{\partial \mu}{\partial x_{3}} B_{1}-\frac{\partial \mu}{\partial x_{1}} B_{3}\right) B_{1} q_{3} \\
& +2\left(\lambda \frac{\partial D_{2}}{\partial x_{3}}-\lambda \frac{\partial D_{3}}{\partial x_{2}}\right) D_{2} q_{3}+2\left(\mu \frac{\partial B_{1}}{\partial x_{3}}-\mu \frac{\partial B_{3}}{\partial x_{1}}\right) B_{1} q_{3} \\
= & -2 q_{3}\left(\frac{\partial \lambda}{\partial x_{2}} D_{3} D_{2}-\frac{\partial \lambda}{\partial x_{3}} D_{2}^{2}\right)-2 q_{3}\left(\frac{\partial \mu}{\partial x_{1}} B_{3} B_{1}-\frac{\partial \mu}{\partial x_{3}} B_{1}^{2}\right) \\
& -2 q_{3} \lambda \frac{\partial D_{3}}{\partial x_{2}} D_{2}+q_{3} \lambda \frac{\partial\left(D_{2}^{2}\right)}{\partial x_{3}}-2 q_{3} \mu \frac{\partial B_{3}}{\partial x_{1}} B_{1}+q_{3} \mu \frac{\partial\left(B_{1}^{2}\right)}{\partial x_{3}}
\end{aligned}
$$

If we integrate by parts their difference in $\Omega \times(0,+\infty)$, denoting by $\nu_{1}, \nu_{2}, \nu_{3}$ the scalar components of $\nu$, we obtain

$$
\begin{aligned}
2 \int_{0}^{T} \int_{\Omega}\left[\left(D_{1} B_{2} q_{3}\right)^{\prime}-\left(D_{2} B_{1} q_{3}\right)^{\prime}\right] \mathrm{d} x \mathrm{~d} t= & \int_{0}^{T} \int_{\Gamma}\left(2 q_{3} \mu B_{3} B_{2} \nu_{2}-q_{3} \mu B_{2}^{2} \nu_{3}+2 q_{3} \lambda D_{1} D_{3} \nu_{1}-q_{3} \lambda D_{1}^{2} \nu_{3}\right. \\
& \left.-q_{3} \mu B_{1}^{2} \nu_{3}+2 q_{3} \mu B_{1} B_{3} \nu_{1}-q_{3} \lambda D_{2}^{2} \nu_{3}+2 q_{3} \lambda D_{2} D_{3} q_{3} \nu_{2}\right) \mathrm{d} \Gamma \mathrm{d} t \\
& +\int_{0}^{T} \int_{\Omega}\left(-2 q_{3} \mu B_{3} \frac{\partial B_{2}}{\partial x_{2}}-2 \frac{\partial q_{3}}{\partial x_{2}} \mu B_{2} B_{3}+\frac{\partial q_{3}}{\partial x_{3}} \mu B_{2}^{2}-2 q_{3} \lambda \frac{\partial D_{1}}{\partial x_{1}} D_{3}\right. \\
& -2 \frac{\partial q_{3}}{\partial x_{1}} \lambda D_{1} D_{3}+\frac{\partial q_{3}}{\partial x_{3}} \lambda D_{1}^{2}+\frac{\partial q_{3}}{\partial x_{3}} \mu B_{1}^{2}-2 q_{3} \mu B_{3} \frac{\partial B_{1}}{\partial x_{1}} \\
& \left.-2 \frac{\partial q_{3}}{\partial x_{1}} \mu B_{1} B_{3}+\frac{\partial q_{3}}{\partial x_{3}} \lambda D_{2}^{2}-2 q_{3} \lambda \frac{\partial D_{2}}{\partial x_{2}} D_{3}-2 \frac{\partial q_{3}}{\partial x_{2}} \lambda D_{2} D_{3}\right) \mathrm{d} x \mathrm{~d} t \\
& +\int_{0}^{T} \int_{\Omega}\left(-q_{3} \frac{\partial \mu}{\partial x_{3}} B_{2}^{2}-q_{3} \frac{\partial \lambda}{\partial x_{3}} D_{1}^{2}-q_{3} \frac{\partial \mu}{\partial x_{3}} B_{1}^{2}-q_{3} \frac{\partial \lambda}{\partial x_{3}} D_{2}^{2}\right) \mathrm{d} x \mathrm{~d} t
\end{aligned}
$$


which may be rewritten as follows:

$$
\begin{aligned}
2\left[\int_{\Omega}\left(D_{1} B_{2} q_{3}-D_{2} B_{1} q_{3}\right) \mathrm{d} x\right]_{0}^{T}= & \int_{0}^{T} \int_{\Gamma}\left\{-q_{3} \nu_{3}\left(\mu B_{1}^{2}+\mu B_{2}^{2}+\lambda D_{1}^{2}+\lambda D_{2}^{2}\right)+2 q_{3} \nu_{2}\left(\mu B_{2} B_{3}+\lambda D_{2} D_{3}\right)\right. \\
& \left.+2 q_{3} \nu_{1}\left(\mu B_{1} B_{3}+\lambda D_{1} D_{3}\right)\right\} \mathrm{d} \Gamma \mathrm{d} t \\
& +\int_{0}^{T} \int_{\Omega}\left\{\frac{\partial q_{3}}{\partial x_{3}}\left(\mu B_{1}^{2}+\mu B_{2}^{2}+\lambda D_{1}^{2}+\lambda D_{2}^{2}\right)-2 q_{3} B_{3}\left(\mu \frac{\partial B_{1}}{\partial x_{1}}+\mu \frac{\partial B_{2}}{\partial x_{2}}\right)\right. \\
& -2 q_{3} D_{3}\left(\lambda \frac{\partial D_{1}}{\partial x_{1}}+\lambda \frac{\partial D_{2}}{\partial x_{2}}\right)-2 \frac{\partial q_{3}}{\partial x_{2}}\left(\lambda D_{2} D_{3}+\mu B_{2} B_{3}\right) \\
& \left.-2 \frac{\partial q_{3}}{\partial x_{1}}\left(\lambda D_{1} D_{3}+\mu B_{1} B_{3}\right)\right\} \mathrm{d} x \mathrm{~d} t \\
& +\int_{0}^{T} \int_{\Omega}\left\{-q_{3} \frac{\partial \mu}{\partial x_{3}}\left(B_{1}^{2}+B_{2}^{2}\right)-q_{3} \frac{\partial \lambda}{\partial x_{3}}\left(D_{1}^{2}+D_{2}^{2}\right)\right\} \mathrm{d} x \mathrm{~d} t .
\end{aligned}
$$

We observe that

$$
\begin{aligned}
\int_{\Omega}-2 q_{3} \mu B_{3}\left(\frac{\partial B_{1}}{\partial x_{1}}+\frac{\partial B_{2}}{\partial x_{2}}\right) \mathrm{d} x & =\int_{\Omega} 2 q_{3} \mu B_{3} \frac{\partial B_{3}}{\partial x_{3}} \mathrm{~d} x=\int_{\Omega} q_{3} \mu \frac{\partial\left(B_{3}^{2}\right)}{\partial x_{3}} \mathrm{~d} x \\
& =\int_{\Gamma} q_{3} \mu B_{3}^{2} \nu_{3} \mathrm{~d} \Gamma-\int_{\Omega} \frac{\partial q_{3}}{\partial x_{3}} \mu B_{3}^{2} \mathrm{~d} x-\int_{\Omega} q_{3} \frac{\partial \mu}{\partial x_{3}} B_{3}^{2} \mathrm{~d} x
\end{aligned}
$$

and analogously

$$
\begin{aligned}
\int_{\Omega}-2 q_{3} \lambda D_{3}\left(\frac{\partial D_{1}}{\partial x_{1}}+\frac{\partial D_{2}}{\partial x_{2}}\right) \mathrm{d} x & =\int_{\Omega} 2 q_{3} \lambda D_{3} \frac{\partial D_{3}}{\partial x_{3}} \mathrm{~d} x=\int_{\Omega} q_{3} \lambda \frac{\partial\left(D_{3}^{2}\right)}{\partial x_{3}} \mathrm{~d} x \\
& =\int_{\Gamma} q_{3} \lambda D_{3}^{2} \nu_{3} \mathrm{~d} \Gamma-\int_{\Omega} \frac{\partial q_{3}}{\partial x_{3}} \lambda D_{3}^{2} \mathrm{~d} x-\int_{\Omega} q_{3} \frac{\partial \lambda}{\partial x_{3}} D_{3}^{2} \mathrm{~d} x .
\end{aligned}
$$

Therefore

$$
\begin{aligned}
{\left[2 \int_{\Omega}\left(D_{1} B_{2} q_{3}-D_{2} B_{1} q_{3}\right) \mathrm{d} x\right]_{0}^{T}=} & \int_{0}^{T} \int_{\Gamma}\left\{-q_{3} \nu_{3}\left(\mu B_{1}^{2}+\mu B_{2}^{2}-\mu B_{3}^{2}+\lambda D_{1}^{2}+\lambda D_{2}^{2}-\lambda D_{3}^{2}\right)\right. \\
& \left.+2 q_{3} \nu_{2}\left(\mu B_{2} B_{3}+\lambda D_{2} D_{3}\right)+2 q_{3} \nu_{1}\left(\mu B_{1} B_{3}+\lambda D_{1} D_{3}\right)\right\} \mathrm{d} \Gamma \mathrm{d} t \\
& +\int_{0}^{T} \int_{\Omega}\left\{\frac{\partial q_{3}}{\partial x_{3}}\left(\mu B_{1}^{2}+\mu B_{2}^{2}-\mu B_{3}^{2}+\lambda D_{1}^{2}+\lambda D_{2}^{2}-\lambda D_{3}^{2}\right)\right. \\
& \left.-2 \frac{\partial q_{3}}{\partial x_{2}}\left(\lambda D_{2} D_{3}+\mu B_{2} B_{3}\right)-2 \frac{\partial q_{3}}{\partial x_{1}}\left(\lambda D_{1} D_{3}+\mu B_{1} B_{3}\right)\right\} \mathrm{d} x \mathrm{~d} t \\
& +\int_{0}^{T} \int_{\Omega}\left(-q_{3} \frac{\partial \mu}{\partial x_{3}}|B|^{2}-q_{3} \frac{\partial \lambda}{\partial x_{3}}|D|^{2}\right) \mathrm{d} x \mathrm{~d} t .
\end{aligned}
$$

By permutation of the indices 1,2,3 we obtain two analogous identities, and summing the three identities we obtain (3.1).

Remark 3.2. Note that the identity (3.1) of Lemma 3.1 is independent of the boundary condition (1.5) and of the initial condition (1.4).

The above identity will be used with $q=m$, the standard multiplier given by

$$
m(x) \equiv x, \quad x \in \Omega .
$$


We now assume that $\Omega$ is strictly star-shaped with respect to the origin, that is

$$
x \cdot \nu(x)>0 \text { for all } x \in \Gamma \text {. }
$$

For further uses we also set

$$
R=\sup _{x \in \Omega}|x|, \quad R_{1}=\max _{x \in \Gamma} \frac{|m|^{2}}{m \cdot \nu} .
$$

To estimate the boundary term of (3.1) in the case $q=m$ we shall use the next estimate.

Lemma 3.3. Assume (3.3). Let $\left(D_{0}, B_{0}\right)$ be an initial pair $\in \mathcal{H}_{1}$ and let $(D, B)$ be the solution of the system (1.1-1.4) with boundary condition (1.5). Then,

$$
(m \cdot \nu)\left(\mu|B|^{2}+\lambda|D|^{2}\right)-2 \lambda(m \cdot D)(\nu \cdot D)-2 \mu(m \cdot B)(\nu \cdot B) \leq R_{1}\left(\mu\left|B_{\tau}\right|^{2}+\lambda\left|D_{\tau}\right|^{2}\right)
$$

on $\Gamma$.

Proof. We use similar arguments than in Lemma 8.21 of [11]:

Denote $D_{\nu}:=D \cdot \nu$ and $B_{\nu}:=B \cdot \nu$, so that

$$
D=D_{\tau}+D_{\nu} \nu, \quad B=B_{\tau}+B_{\nu} \nu
$$

The left-hand side of the estimate that we want to prove can be rewritten as

$$
(m \cdot \nu)\left(\mu\left|B_{\tau}\right|^{2}+\lambda\left|D_{\tau}\right|^{2}-\mu B_{\nu}^{2}-\lambda D_{\nu}^{2}\right)-2 \lambda\left(m_{\tau} \cdot D_{\tau}\right) D_{\nu}-2 \mu\left(m_{\tau} \cdot B_{\tau}\right) B_{\nu}
$$

where with $m_{\tau}$ we denote the tangential component of $m$.

Since $\Omega$ is strictly star-shaped we can estimate

$$
-2 \lambda\left(m_{\tau} \cdot D_{\tau}\right) D_{\nu} \leq \lambda(m \cdot \nu) D_{\nu}^{2}+\lambda \frac{\left(m_{\tau} \cdot D_{\tau}\right)^{2}}{m \cdot \nu}
$$

and, analogously,

$$
-2 \mu\left(m_{\tau} \cdot B_{\tau}\right) B_{\nu} \leq \mu(m \cdot \nu) B_{\nu}^{2}+\mu \frac{\left(m_{\tau} \cdot B_{\tau}\right)^{2}}{m \cdot \nu}
$$

Then,

$$
\begin{gathered}
(m \cdot \nu)\left(\mu|B|^{2}+\lambda|D|^{2}\right)-2 \lambda(m \cdot D)(\nu \cdot D)-2 \mu(m \cdot B)(\nu \cdot B) \leq(m \cdot \nu)\left(\mu\left|B_{\tau}\right|^{2}+\lambda\left|D_{\tau}\right|^{2}\right) \\
+\frac{\lambda\left(m_{\tau} \cdot D_{\tau}\right)^{2}+\mu\left(m_{\tau} \cdot B_{\tau}\right)^{2}}{m \cdot \nu} \\
\leq(m \cdot \nu)\left(\mu\left|B_{\tau}\right|^{2}+\lambda\left|D_{\tau}\right|^{2}\right)+\frac{\left|m_{\tau}\right|^{2}}{m \cdot \nu}\left(\lambda\left|D_{\tau}\right|^{2}+\mu\left|B_{\tau}\right|^{2}\right) .
\end{gathered}
$$

Note that

$$
\left[(m \cdot \nu)+\frac{\left|m_{\tau}\right|^{2}}{m \cdot \nu}\right]\left(\mu\left|B_{\tau}\right|^{2}+\lambda\left|D_{\tau}\right|^{2}\right)=\frac{|m|^{2}}{m \cdot \nu}\left(\mu\left|B_{\tau}\right|^{2}+\lambda\left|D_{\tau}\right|^{2}\right),
$$

and so, recalling the definition of $R_{1}$, the requested estimate is proved. 


\section{Stabilization Result in the linear Case}

In the full section we assume that $g$ is in the form. $g(x, D)=\alpha(x) D$, with $\alpha \in C^{1}(\Gamma)$.

The results of section 3 allows to prove the following:

Theorem 4.1. Assume (3.3) and consider $\lambda, \mu \in C^{1}(\bar{\Omega} \times[0,+\infty)), \alpha \in C^{1}(\Gamma)$ satisfying (2.11, 2.12, 2.17) as well as the following hypotheses:

$$
\begin{aligned}
& \alpha(x)>0, \quad \forall x \in \Gamma, \\
& \mu-m \cdot \nabla \mu-\frac{R_{1}}{2 L}\left(\frac{1}{\min _{\Gamma} \alpha}+\max _{\Gamma} \alpha\right) \frac{\partial \mu}{\partial t} \geq c_{0} \mu, \forall x \in \Omega, t \in(0,+\infty), \\
& \lambda-m \cdot \nabla \lambda-\frac{R_{1}}{2 L}\left(\frac{1}{\min _{\Gamma} \alpha}+\max _{\Gamma} \alpha\right) \frac{\partial \lambda}{\partial t} \geq c_{0} \lambda, \forall x \in \Omega, t \in(0,+\infty),
\end{aligned}
$$

where $c_{0}$ is a suitable positive constant. Then, for any given $\left(D_{0}, B_{0}\right) \in \mathcal{H}_{1}$ the solution of (1.1-1.4) and (1.5) with $g(x, D)=\alpha(x) D$ satisfies the estimate

$$
\mathcal{E}(t) \leq \mathcal{E}(0) \mathrm{e}^{1-c^{*} t}, \quad \forall t \geq 0
$$

with

$$
c^{*}=\frac{2 c_{0} \min \{\inf \lambda, \inf \mu\}}{2 R+R_{1}\left(\max _{\Gamma} \alpha+\frac{1}{\min _{\Gamma} \alpha}\right)} .
$$

Remark 4.2. Hypotheses (4.2) and (4.3) are growth assumptions on the functions $\lambda, \mu$. They hold true, for example, if these functions are non-increasing in time and such that $x \cdot \nabla \lambda(x, t) \leq 0$ and $x \cdot \nabla \mu(x, t) \leq 0$ in $\Omega$.

Proof of Theorem 4.1. First of all note that

$$
\left|2 \int_{\Omega}(D \times B) \cdot m \mathrm{~d} x\right| \leq 2 R \int_{\Omega}|D||B| \mathrm{d} x \leq R \int_{\Omega}\left(|D|^{2}+|B|^{2}\right) \mathrm{d} x \leq \frac{R}{L} \int_{\Omega}\left(\lambda|D|^{2}+\mu|B|^{2}\right) \mathrm{d} x .
$$

Formula (3.1), rewritten for $q(x)=m(x)$, becomes

$$
\begin{aligned}
{\left[2 \int_{\Omega}(D \times B) \cdot m \mathrm{~d} x\right]_{S}^{T}=} & \int_{S}^{T} \int_{\Gamma}\left\{-\left(\lambda|D|^{2}+\mu|B|^{2}\right)(m \cdot \nu)\right. \\
& +2 \mu(m \cdot B)(\nu \cdot B)+2 \lambda(m \cdot D)(\nu \cdot D)\} \mathrm{d} \Gamma \mathrm{d} t \\
& +\int_{S}^{T} \int_{\Omega}\left(\lambda|D|^{2}+\mu|B|^{2}\right) \mathrm{d} x \mathrm{~d} t \\
& +\int_{S}^{T} \int_{\Omega}\left[-(m \cdot \nabla \lambda)|D|^{2}-(m \cdot \nabla \mu)|B|^{2}\right] \mathrm{d} x \mathrm{~d} t
\end{aligned}
$$

By (4.6), using Lemma 3.3 and the estimate (4.5), it follows

$$
\begin{aligned}
\int_{S}^{T} \int_{\Omega}\left(\lambda|D|^{2}+\mu|B|^{2}\right) \mathrm{d} x \mathrm{~d} t \leq \frac{2 R}{L}[\mathcal{E}(T)+\mathcal{E}(S)]+\int_{S}^{T} \int_{\Gamma} & R_{1}\left(\mu\left|B_{\tau}\right|^{2}+\lambda\left|D_{\tau}\right|^{2}\right) \mathrm{d} \Gamma \mathrm{d} t \\
& \quad+\int_{S}^{T} \int_{\Omega}\left[(m \cdot \nabla \lambda)|D|^{2}+(m \cdot \nabla \mu)|B|^{2}\right] \mathrm{d} x \mathrm{~d} t
\end{aligned}
$$


and then,

$$
\begin{aligned}
& \int_{S}^{T} \int_{\Omega}\left[(\mu-m \cdot \nabla \mu)|B|^{2}+(\lambda-m \cdot \nabla \lambda)|D|^{2}\right] \mathrm{d} x \mathrm{~d} t \\
& \leq \frac{2 R}{L}[\mathcal{E}(S)+\mathcal{E}(T)]+\frac{R_{1}}{L} \int_{S}^{T} \int_{\Gamma} \mu \lambda\left|D_{\tau}\right|^{2} \mathrm{~d} \Gamma \mathrm{d} t+\frac{R_{1}}{L} \int_{S}^{T} \int_{\Gamma} \mu \lambda\left|B_{\tau}\right|^{2} \mathrm{~d} \Gamma \mathrm{d} t \\
& \leq \frac{2 R}{L}[\mathcal{E}(S)+\mathcal{E}(T)]+\frac{R_{1}}{L} \frac{1}{\min _{\Gamma} \alpha} \int_{S}^{T} \int_{\Gamma} \alpha \mu \lambda\left|D_{\tau}\right|^{2} \mathrm{~d} x \mathrm{~d} t \\
& \quad+\frac{R_{1}}{L} \max _{\Gamma} \alpha \int_{S}^{T} \int_{\Gamma} \frac{1}{\alpha} \mu \lambda\left|B_{\tau}\right|^{2} \mathrm{~d} x \mathrm{~d} t .
\end{aligned}
$$

Therefore, recalling (2.15) and (2.16),

$$
\begin{aligned}
& \int_{S}^{T} \int_{\Omega}\left[(\mu-m \cdot \nabla \mu)|B|^{2}+(\lambda-m \cdot \nabla \lambda)|D|^{2}\right] \mathrm{d} x \mathrm{~d} t \leq \frac{2 R}{L}[\mathcal{E}(S)+\mathcal{E}(T)] \\
&+\frac{R_{1}}{L} \frac{1}{\min _{\Gamma} \alpha}[\mathcal{E}(S)-\mathcal{E}(T)]+\frac{R_{1}}{2 L} \frac{1}{\min _{\Gamma} \alpha} \int_{S}^{T} \int_{\Omega}\left\{\frac{\partial \lambda}{\partial t}|D|^{2}+\frac{\partial \mu}{\partial t}|B|^{2}\right\} \mathrm{d} x \mathrm{~d} t \\
&+\frac{R_{1}}{L} \max _{\Gamma} \alpha[\mathcal{E}(S)-\mathcal{E}(T)]+\frac{R_{1}}{2 L} \max _{\Gamma} \alpha \int_{S}^{T} \int_{\Omega}\left\{\frac{\partial \lambda}{\partial t}|D|^{2}+\frac{\partial \mu}{\partial t}|B|^{2}\right\} \mathrm{d} x \mathrm{~d} t
\end{aligned}
$$

By the assumptions (4.2) and (4.3), the above estimate may be transformed into

$$
2 c_{0} \int_{S}^{T} \mathcal{E}(t) \mathrm{d} t \quad \leq \frac{2 R}{L}[\mathcal{E}(S)+\mathcal{E}(T)]+\frac{R_{1}}{L}\left(\max _{\Gamma} \alpha+\frac{1}{\min _{\Gamma} \alpha}\right)[\mathcal{E}(S)-\mathcal{E}(T)] .
$$

Now, we note that

$$
\frac{2 R}{L}-\frac{R_{1}}{L}\left(\max _{\Gamma} \alpha+\frac{1}{\min _{\Gamma} \alpha}\right) \leq 0
$$

Indeed, by Young's inequality we have

$$
2 R \leq R \alpha+\frac{R}{\alpha} \leq R \max _{\Gamma} \alpha+\frac{R}{\min _{\Gamma} \alpha} \leq R_{1} \max _{\Gamma} \alpha+\frac{R_{1}}{\min _{\Gamma} \alpha}
$$

because $R_{1} \geq R$.

Then the above estimate in (4.9) yields

$$
2 c_{0} \int_{S}^{T} \mathcal{E}(t) \mathrm{d} t \leq \frac{2 R}{L} \mathcal{E}(S)+\frac{R_{1}}{L}\left(\max _{\Gamma} \alpha+\frac{1}{\min _{\Gamma} \alpha}\right) \mathcal{E}(S)
$$

from which follows, taking the limit for $T \rightarrow+\infty$

$$
2 c_{0} \int_{S}^{+\infty} \mathcal{E}(t) \mathrm{d} t \leq \frac{1}{L}\left(2 R+R_{1} \max _{\Gamma} \alpha+R_{1} \frac{1}{\min _{\Gamma} \alpha}\right) \mathcal{E}(S) .
$$

Since $\mathcal{E}(\cdot)$ is positive and non-increasing, the stabilization estimate (4.4) follows by a well-known argument (see e.g. Th. 9.1 of [11]). 


\section{Stabilization Result in the nOnLinear CASE}

In this section we consider our system (1.1-1.3) and (1.4) with nonlinear feedbacks $(1.5)$ as in $[5,15]$.

Here the difference with $[5,15]$ is that we cannot use Liu's principle since our system is not reversible due to the time dependence of our coefficients $\lambda$ and $\mu$ so we use a direct method based on the identity with multiplier (3.1) to estimate the energy by boundary terms.

These boundary terms are then estimated by a function of the energy using the property of $g$ and, as in [5,15], using a new integral inequality we deduce decay rates of the energy.

Theorem 5.1. Assume (3.3) and consider $\lambda, \mu \in C^{1}(\bar{\Omega} \times[0,+\infty))$ satisfying the hypotheses (2.11, 2.12, 2.17) and

$$
\begin{aligned}
& \mu-m \cdot \nabla \mu-d \frac{\partial \mu}{\partial t} \geq c_{1} \mu, \forall x \in \Omega, t \in(0,+\infty), \\
& \lambda-m \cdot \nabla \lambda-d \frac{\partial \lambda}{\partial t} \geq c_{1} \lambda, \forall x \in \Omega, t \in(0,+\infty),
\end{aligned}
$$

for some positive constant $c_{1}$ where for shorthness we have set $d=\frac{\left(1+4 M^{2}\right) R_{1}}{2 m_{1} L}$.

Assume that $g$ satisfies the assumptions (1.6) to (1.8), as well as

$$
|E|^{2}+|g(x, E)|^{2} \leq G(g(x, E) \cdot E), \forall|E| \leq 1, x \in \Gamma,
$$

for some concave strictly increasing function $G:[0, \infty) \rightarrow[0, \infty)$ such that $G(0)=0$.

Then, for any given $\left(D_{0}, B_{0}\right) \in \mathcal{H}_{1}$ the solution of $(1.1)-(1.4)$ and $(1.5)$ satisfies the estimate

$$
\mathcal{E}(t) \leq c_{3} G\left(\frac{\psi^{-1}\left(c_{2} t\right)}{c_{2} T^{2}|\Gamma| t}\right), \forall t \geq T_{1}
$$

for $T>0$ large enough and some positive constants $c_{2}, c_{3}, T_{1}$ depending on $T, \mathcal{E}(0)$ and $|\Gamma|$ and finally

$$
\phi(t)=T|\Gamma| L^{2} G^{-1}\left(\frac{t}{c_{3}}\right), \psi(t)=\int_{t}^{1} \frac{1}{\phi(s)} \mathrm{d} s, \forall t>0 .
$$

Proof. We start from (4.7) which is valid for any boundary conditions and remark that from (1.5) we get

$$
\left|B_{\tau}\right|=|g(\cdot, D \times \nu) \times \nu| \leq|g(\cdot, D \times \nu)|,
$$

therefore (4.7) becomes (for $0 \leq S<T$, compare with (4.8))

$$
\begin{aligned}
\int_{S}^{T} \int_{\Omega}\left[(\mu-m \cdot \nabla \mu)|B|^{2}\right. & \left.+(\lambda-m \cdot \nabla \lambda)|D|^{2}\right] \mathrm{d} x \mathrm{~d} t \leq \frac{2 R}{L}[\mathcal{E}(S)+\mathcal{E}(T)] \\
& +R_{1} \int_{S}^{T} \int_{\Gamma}\left(\mu\left|B_{\tau}\right|^{2}+\lambda\left|D_{\tau}\right|^{2}\right) \mathrm{d} \Gamma \mathrm{d} t \leq \frac{4 R}{L} \mathcal{E}(S) \\
& +\frac{R_{1}}{L} \int_{S}^{T} \int_{\Gamma} \lambda \mu\left(|g(x, D \times \nu)|^{2}+|D \times \nu|^{2}\right) \mathrm{d} \Gamma \mathrm{d} t
\end{aligned}
$$

We now estimate the second term of this right-hand side as follows:

Introduce

$$
\begin{aligned}
& \Sigma_{S T}=\Gamma \times(S, T), \\
& \Sigma_{S T}^{+}=\left\{(x, t) \in \Sigma_{S T}|| D(x, t) \times \nu(x) \mid>1\right\} \\
& \Sigma_{S T}^{-}=\left\{(x, t) \in \Sigma_{S T}|| D(x, t) \times \nu(x) \mid \leq 1\right\} .
\end{aligned}
$$


Then by the conditions (1.8) and (1.9) we first have

$$
\begin{aligned}
\int_{\Sigma_{S T}^{+}} \lambda \mu\left(|g(x, D \times \nu)|^{2}+|D \times \nu|^{2}\right) \mathrm{d} \Gamma \mathrm{d} t & \leq \frac{1+4 M^{2}}{m_{1}} \int_{\Sigma_{S T}^{+}} \lambda \mu g(x, D \times \nu) \cdot D \times \nu \mathrm{d} \Gamma \mathrm{d} t \\
& \leq \frac{1+4 M^{2}}{m_{1}} \int_{\Sigma_{S T}} \lambda \mu g(x, D \times \nu) \cdot D \times \nu \mathrm{d} \Gamma \mathrm{d} t
\end{aligned}
$$

since the conditions (1.6) and (1.7) imply that $g(\cdot, E) \cdot E \geq 0$.

By the identity (2.14), we arrive at

$$
\begin{aligned}
\int_{\Sigma_{S T}^{+}} \lambda \mu\left(|g(x, D \times \nu)|^{2}+|D \times \nu|^{2}\right) \mathrm{d} \Gamma \mathrm{d} t \leq & \frac{1+4 M^{2}}{m_{1}}(\mathcal{E}(S)-\mathcal{E}(T)) \\
& +\frac{1+4 M^{2}}{2 m_{1}} \int_{S}^{T} \int_{\Omega}\left\{\frac{\partial \lambda}{\partial t}|D|^{2}+\frac{\partial \mu}{\partial t}|B|^{2}\right\} \mathrm{d} x \mathrm{~d} t .
\end{aligned}
$$

For the estimation of the integral on $\Sigma_{S T}^{-}$, we remark that the assumption (5.3) directly yields

$$
\begin{aligned}
\int_{\Sigma_{S T}^{-}} \lambda \mu\left(|g(x, D \times \nu)|^{2}+|D \times \nu|^{2}\right) \mathrm{d} \Gamma \mathrm{d} t & \leq \int_{\Sigma_{S T}^{-}} G(g(x, D \times \nu) \cdot D \times \nu) \lambda \mu \mathrm{d} \Gamma \mathrm{d} t \\
& \leq \int_{\Sigma_{S T}} G(g(x, D \times \nu) \cdot D \times \nu) \lambda \mu \mathrm{d} \Gamma \mathrm{d} t
\end{aligned}
$$

since the properties of $G$ implies that $G(s) \geq 0$, for all $s \geq 0$.

Using Jensen's inequality we obtain

$$
\int_{\Sigma_{S T}^{-}} \lambda \mu\left(|g(x, D \times \nu)|^{2}+|D \times \nu|^{2}\right) \mathrm{d} \Gamma \mathrm{d} t \leq m_{S T} G\left(\frac{1}{m_{S T}} \int_{\Sigma_{S T}} \lambda \mu g(x, D \times \nu) \cdot D \times \nu \mathrm{d} \Gamma \mathrm{d} t\right),
$$

where $m_{S T}=\int_{\Sigma_{S T}} \lambda(x, t) \mu(x, t) \mathrm{d} \Gamma(x) \mathrm{d} t$. Note that the property (2.17) implies that

$$
m_{S T} \leq \int_{\Sigma_{S T}} \lambda(x, 0) \mu(x, 0) \mathrm{d} \Gamma(x) \mathrm{d} t=(T-S) \int_{\Gamma} \lambda(x, 0) \mu(x, 0) \mathrm{d} \Gamma(x)=(T-S) c_{4},
$$

while (2.11) yields

$$
m_{S T} \geq(T-S)|\Gamma| L^{2} .
$$

Using again the identity $(2.14)$, the property (2.17) as well as the increaseness of $G$, we conclude from the three above inequalities that

$$
\int_{\Sigma_{S T}^{-}} \lambda \mu\left(|g(x, D \times \nu)|^{2}+|D \times \nu|^{2}\right) \mathrm{d} \Gamma \mathrm{d} t \leq(T-S) c_{4} G\left(\frac{\mathcal{E}(S)-\mathcal{E}(T)}{(T-S)|\Gamma| L^{2}}\right) .
$$

The estimates (5.6) and (5.7) in (5.5) yield

$$
\begin{array}{r}
\int_{S}^{T} \int_{\Omega}\left[(\mu-m \cdot \nabla \mu)|B|^{2}+(\lambda-m \cdot \nabla \lambda)|D|^{2}-d\left\{\frac{\partial \lambda}{\partial t}|D|^{2}+\frac{\partial \mu}{\partial t}|B|^{2}\right\}\right] \mathrm{d} x \mathrm{~d} t \leq \frac{4 R}{L} \mathcal{E}(S) \\
+\frac{\left(1+4 M^{2}\right) R_{1}}{m_{1} L}(\mathcal{E}(S)-\mathcal{E}(T))+\frac{(T-S) c_{4} R_{1}}{L} G\left(\frac{\mathcal{E}(S)-\mathcal{E}(T)}{(T-S)|\Gamma| L^{2}}\right)
\end{array}
$$


By the assumptions (5.1) and (5.2) we obtain

$$
2 c_{1} \int_{S}^{T} \mathcal{E}(t) \mathrm{d} t \leq \frac{4 R}{L} \mathcal{E}(S)+\frac{\left(1+4 M^{2}\right) R_{1}}{m_{1} L}(\mathcal{E}(S)-\mathcal{E}(T))+\frac{(T-S) c_{4} R_{1}}{L} G\left(\frac{\mathcal{E}(S)-\mathcal{E}(T)}{(T-S)|\Gamma| L^{2}}\right)
$$

Using the nonincreaseness of the energy the above estimate becomes

$$
2 c_{1}(T-S) \mathcal{E}(T) \leq \frac{4 R}{L} \mathcal{E}(S)+\frac{\left(1+4 M^{2}\right) R_{1}}{m_{1} L}(\mathcal{E}(S)-\mathcal{E}(T))+\frac{(T-S) c_{4} R_{1}}{L} G\left(\frac{\mathcal{E}(S)-\mathcal{E}(T)}{(T-S)|\Gamma| L^{2}}\right)
$$

Now the trivial identity $\mathcal{E}(S)=\mathcal{E}(T)+(\mathcal{E}(S)-\mathcal{E}(T))$ allows to transform the above estimate into

$$
\begin{aligned}
2 c_{1}(T-S) \mathcal{E}(S) \leq & \frac{4 R}{L} \mathcal{E}(S)+\left(2 c_{1}(T-S)+\frac{\left(1+4 M^{2}\right) R_{1}}{m_{1} L}\right)(\mathcal{E}(S)-\mathcal{E}(T)) \\
& +\frac{(T-S) c_{4} R_{1}}{L} G\left(\frac{\mathcal{E}(S)-\mathcal{E}(T)}{(T-S)|\Gamma| L^{2}}\right)
\end{aligned}
$$

Finally the nonincreaseness of the energy implies that

$$
\frac{\mathcal{E}(S)-\mathcal{E}(T)}{(T-S)|\Gamma| L^{2}} \leq \frac{\mathcal{E}(S)}{(T-S)|\Gamma| L^{2}} \leq \frac{\mathcal{E}(0)}{(T-S)|\Gamma| L^{2}},
$$

and the concavity of $G$ yields a constant $c_{5}$ (depending continuously on $T-S, \mathcal{E}(0),|\Gamma|$ and $L^{2}$ ) such that

$$
\frac{\mathcal{E}(S)-\mathcal{E}(T)}{(T-S)|\Gamma| L^{2}} \leq c_{5} G\left(\frac{\mathcal{E}(S)-\mathcal{E}(T)}{(T-S)|\Gamma| L^{2}}\right) .
$$

This estimate in (5.8) allows to conclude that there exists a positive constant $c_{6}$ (depending continuously on $T-S, \mathcal{E}(0),|\Gamma|$ and $\left.L^{2}\right)$ such that

$$
\left(2 c_{1}(T-S)-\frac{4 R}{L}\right) \mathcal{E}(S) \leq c_{6} G\left(\frac{\mathcal{E}(S)-\mathcal{E}(T)}{(T-S)|\Gamma| L^{2}}\right) .
$$

Choosing $T-S$ large enough such that $2 c_{1}(T-S)-\frac{4 R}{L}>1 / 2$, we have found that

$$
\mathcal{E}(S) \leq 2 c_{6} G\left(\frac{\mathcal{E}(S)-\mathcal{E}(T)}{(T-S)|\Gamma| L^{2}}\right)
$$

In this estimate we now substitute $S$ into $t$ and $T$ into $t+T$.

Therefore we have proved that for $T$ large enough and all $t \geq 0$ we have

$$
\mathcal{E}(t) \leq c_{3} G\left(\frac{\mathcal{E}(t)-\mathcal{E}(t+T)}{T|\Gamma| L^{2}}\right)=\phi^{-1}(\mathcal{E}(t)-\mathcal{E}(t+T)) .
$$

We then conclude by Lemma 5.1 and Theorem 1.2 of [5].

Remark 5.2. Examples of functions $g$ leading to an explicit decay rate $(5.4)$ are given in $[5,15]$. Let us notice that exponential, polynomial or logarithmic decays are available for appropriate feedbacks.

Remark 5.3. In the time independent case, the use of microlocal analysis technique in $[5,15]$ allows to leave the condition that the domain is strictly star shaped with respect to a point, as far as we know we cannot use this technique in the time dependent case. Since we here use the multiplier technique the strictly star 
shaped condition is quite natural. On the other hand a careful analysis of the results from $[5,15]$ shows that the condition

$$
-m \cdot \nabla \mu \geq 0,-m \cdot \nabla \lambda \geq 0, \forall x \in \Omega,
$$

imposed in $[5,15]$ may be replaced by the weaker assumptions $(5.1)$ and $(5.2)$.

The present work started during the conference "Control and stabilization of PDE", Cortona, Italy, May 21-26, 2001. We then thank the organizers for their invitation.

\section{REFERENCES}

[1] H. Barucq and B. Hanouzet, Étude asymptotique du système de Maxwell avec la condition aux limites absorbante de SilverMüller II. C. R. Acad. Sci. Paris Sér. I Math. 316 (1993) 1019-1024.

[2] C. Castro and E. Zuazua, Localization of waves in $1-d$ highly heterogeneous media. Arch. Rational Mech. Anal. 164 (2002) 39-72.

[3] M.G. Crandall and A. Pazy, Nonlinear evolution equations in Banach spaces. Israel J. Math. 11 (1972) 57-94.

[4] R. Dautray and J.L. Lions, Mathematical Analysis and Numerical Methods for Science and Technology. Springer-Verlag, Vol. 3 (1990), Vol. 5 (1992).

[5] M. Eller, J.E. Lagnese and S. Nicaise, Decay rates for solutions of a Maxwell system with nonlinear boundary damping. Comp. Appl. Math. 21 (2002) 135-165.

[6] L.C. Evans, Nonlinear evolution equations in an arbitrary Banach space. Israel J. Math. 26 (1977) 1-42.

[7] P. Grisvard, Elliptic problems in nonsmooth Domains. Pitman, Boston, Monogr. Stud. Math. 21 (1985).

[8] T. Kato, Nonlinear semigroups and evolution equations. J. Math. Soc. Japan 19 (1967) 508-520.

[9] T. Kato, Linear and quasilinear equations of evolution of hyperbolic type, CIME, II Ciclo. Cortona (1976) 125-191.

[10] T. Kato, Abstract differential equations and nonlinear mixed problems. Accademia Nazionale dei Lincei, Scuola Normale Superiore, Lezione Fermiane, Pisa (1985).

[11] V. Komornik, Exact Controllability and Stabilization. The Multiplier Method. Masson-John Wiley, Collection RMA Paris 36 (1994).

[12] V. Komornik, Boundary stabilization, observation and control of Maxwell's equations. Panamer. Math. J. 4 (1994) 47-61.

[13] J.E. Lagnese, Exact controllability of Maxwell's equations in a general region. SIAM J. Control Optim. 27 (1989) 374-388.

[14] C.-Y. Lin, Time-dependent nonlinear evolution equations. Differential Integral Equations 15 (2002) 257-270.

[15] S. Nicaise, M. Eller and J.E. Lagnese, Stabilization of heterogeneous Maxwell's equations by nonlinear boundary feedbacks. EJDE 2002 (2002) 1-26.

[16] S. Nicaise, Exact boundary controllability of Maxwell's equations in heteregeneous media and an application to an inverse source problem. SIAM J. Control Optim. 38 (2000) 1145-1170.

[17] L. Paquet, Problèmes mixtes pour le système de Maxwell. Ann. Fac. Sci. Toulouse Math. 4 (1982) 103-141.

[18] A. Pazy, Semigroups of linear operators and applications to partial differential equations. Springer-Verlag,, Appl. Math. Sci. 44 (1983).

[19] K.D. Phung, Contrôle et stabilisation d'ondes électromagnétiques. ESAIM: COCV 5 (2000) 87-137.

[20] C. Pignotti, Observability and controllability of Maxwell's equations. Rend. Mat. Appl. 19 (1999) 523-546. 